\title{
Ovarian cysts disappear after 14-day oral regimen of Korean red ginseng extract in letrozole-induced polycystic ovarian syndrome
}

\author{
Niknaz Moradi, PharmD', Sepideh Arbabi Bidgoli, PharmD, PhD², Shahla Chaichian, MD \\ ${ }^{1}$ Faculty of Pharmacy and Pharmaceutical Sciences, ${ }^{2}$ Department of Toxicology and Pharmacology, Faculty of Pharmacy and Pharmaceutical Sciences, \\ ${ }^{3}$ Department of Gynecology and Obstetrics, Faculty of Medicine, Islamic Azad University, Tehran Medical Sciences University, Tehran, Iran
}

\section{Objective}

Hormonal and inflammatory mechanisms are involved in the pathogenesis of polycystic ovarian syndrome (PCOS), which is a prevalent metabolic disorder among women of reproductive age. We aimed to evaluate the comparative efficiency of short-term oral administration of Korean red ginseng extract (KRGE) and the standard treatment on PCOS by focusing on the histopathological parameters and serum levels of luteinizing hormone (LH), folliclestimulating hormone (FSH), testosterone, and nuclear factor kappa B (NF-kB).

\section{Methods}

A PCOS rat model was established by oral gavage of letrozole $(1 \mathrm{mg} / \mathrm{kg})$ for 21 days. The serum levels of LH, FSH, testosterone, and NF-кB were measured, and the morphological features and differences of the ovaries were examined in each group using a light microscope before and after 14 days of treatment with oral regimens-KRGE, oral contraceptives (OCPs), KRGE+OCPs, and carboxymethyl cellulose (CMC).

\section{Results}

OCPs alone could not normalize the mean ovarian weights of PCOS rats despite the 14-day oral regimen, but they were more effective in reducing the number and size of cysts compared to others. KRGE alone and in combination with OCPs was effective in normalizing abnormal ovarian weights, decreasing LH serum levels, and dissipating the ovarian cysts in PCOS rats. However, when combined with the standard regimen, KRGE showed additional therapeutic effects by efficiently reducing serum testosterone and NF-KB levels.

\section{Conclusion}

Our necropsy and histopathological evidence suggests the efficacy of KRGE as a novel integrative medicine against abnormal multiple follicular cysts. However, antiandrogenic and anti-inflammatory effects were only seen in animals that were administered a combination of KRGE and the standard regimen.

Keywords: Ovarian cysts; Polycystic ovary syndrome; Panax; Luteinizing hormone; NF-kappa B

\section{Introduction}

Worldwide, polycystic ovarian syndrome (PCOS) is the most common heterogeneous endocrine condition and is prevalent in 6-20\% of women of reproductive age [1]. Ovulatory dysfunction, androgen excess, menstrual irregularities [2], infertility, and clinical evidence of hyperandrogenism are the most important symptoms of PCOS that permanently reduce the quality of life of patients [3]. Other than these hormonal disorders, risks such as cardiovascular disorders, dyslipidemia, obesity, and type 2 diabetes mellitus complicate this syn-
Received: 2020.04.12. Revised: 2020.07.27. Accepted: 2020.09.06. Corresponding author: Sepideh Arbabi Bidgoli, PharmD, PhD Department of Toxicology and Pharmacology, Islamic Azad University, Tehran Medical Sciences University, Shariati St., Tehran 19395/1495, Iran

E-mail: arbabi@iaups.ac.ir

https://orcid.org/0000-0003-4830-8680

Articles published in Obstet Gynecol Sci are open-access, distributed under the terms of the Creative Commons Attribution Non-Commercial License (http://creativecommons. org/licenses/by-nc/3.0/) which permits unrestricted non-commercial use, distribution, and reproduction in any medium, provided the original work is properly cited.

Copyright $\odot 2021$ Korean Society of Obstetrics and Gynecology 


\section{Obstetrics \& Gynecology Science}

Niknaz Moradi, et al. KRGE in PCOs

drome further [4], especially when combined with the psychological effects of PCOS, such as anxiety and depression [5], thus worsening the quality of life of patients [6].

Other than the role of environmental toxicants with endocrine-disrupting properties in the incidence of PCOS [7], it has also been suggested that one's inflammatory background plays a role, which is closely related to insulin resistance [8] and oxidative stress [9]. In fact, the serum levels of many inflammatory mediators such as C-reactive protein; white blood cells [9]; interleukins (ILs) such as IL-1, IL-6, and IL-18 [10]; and tumor necrosis factor-alpha could be significantly increased in patients diagnosed with PCOS $[11,12]$. However, out of the mentioned inflammatory factors, the role of nuclear factor kappa B (NF-kB), one of the most studied key factors in inflammatory pathways, has been poorly understood in the pathogenesis and prognosis of PCOS.

In this context, many limitations exist with regard to the prevention, protection, or definite treatment of PCOS, thus increasing the public's concerns toward alternative medications. Korean red ginseng is one of the most popular herbal remedies in other disease models and is known to have certain anti-inflammatory [13] and antioxidant effects [14] through activation of the NF-kB pathway [15]. However, the estrogenic effects of Korean red ginseng extract (KRGE) is still conflicting based on in vitro, in vivo [16] and clinical evaluations of sexual disorders [17]. One study in 2011 demonstrated how the ovarian morphology of rats and nerve growth factor levels were almost normalized in the regimen that combined KRGE and estradiol valerate. In this study, KRGE lowered the numbers of antral follicles and increased the number of corpora lutea in treated ovaries; this group of researchers was the first to suggest the beneficial effect of KRGE in the treatment of PCOS [17]. Based on this report, we aimed to determine whether oral administration of KRGE can alter the serum levels of PCOS-related prognostic biomarkers and normalize the rat ovaries against PCOS in the letrozole (a nonsteroidal aromatase inhibitor) model. We also aimed to investigate the anti-inflammatory effects of KRGE monotherapy and its combination with a standard oral contraceptive $(\mathrm{OCP})$ regimen by evaluating the ovarian morphology and determining the serum levels of NF-kB, testosterone, and gonadotropins.

\section{Materials and methods}

\section{Animals}

Female Wistar rats ( $n=25)$, weighing 140 to $160 \mathrm{~g}$, with regular 4-day estrous cycles were purchased from Pasteur Institute of Iran. The rats were quarantined and acclimated for 10 days prior to use. The Ethics Committee of Islamic Azad University, Tehran Medical Sciences University (IAUTMU), approved the protocols of this study under the number IR.IAU. TMU.REC.1397.313, and the animals were cared for in accordance with the Guidelines for Animal Experiments of the Ministry of Health and Medical Education of Iran for the Care and Use of Laboratory Animals and the CCAC Guidelines for Care and Use of Experimental Animals. Rats were caged under standard laboratory indoor conditions with a 12-hour light/dark cycle at room temperature $\left(23 \pm 2^{\circ} \mathrm{C}\right)$ and $20 \%$ relative humidity with free access to tap water and a standard diet for all treatment groups. They were given free access to the standard pellet diet and drinking water ad libitum, but daily food and water intake were calculated until the end of the study. The cage cleaning schedule, air filtration, recirculation, health checks, and facility maintenance were carried out in accordance with the standard operating procedures and were recorded.

\section{Reagents}

KRGE was manufactured by Korea Ginseng Corporation (Seoul, Korea) from 6-year-old Korean RG plants (P. Ginseng). KRGE was purchased in 2018 by the main investigator from the local market of Seoul, Korea as 10 Stick Packs to yield 3,000 mg KRGE. According to the company's descriptions during the visit of the main site in October 2018, the KRG roots were extracted by steaming fresh Korean ginseng, which was previously harvested on-site, at $90-100^{\circ} \mathrm{C}$ for 3 hours. After drying the plant material at $50-80^{\circ} \mathrm{C}, \mathrm{KRGE}$ was extracted by circulating hot water through the dried plant material three times at $85-90^{\circ} \mathrm{C}$ for 8 hours. We diluted this high-quality KRGE each time with purified water before oral gavage to the rats. According to the literature and company information, the present KRGE sample contained three major ginsenosides, $\mathrm{Rg} 1+\mathrm{Rb} 1+\mathrm{Rg} 3$, in an $11.6 \mathrm{mg} / 10 \mathrm{~mL}$ concentration [18]. Carboxymethyl cellulose (CMC) was obtained from a local branch of the Merck Company in Tehran and diluted with purified water each time before gavage to rats.

Other pharmaceutical-grade active pharmaceutical ingredi- 


\section{Obstetrics \& Gynecology Science}

Vol. 64, No. 3, 2021

ents-letrozole (LET), ethinylestradiol (E), and levonorgestrel (L)-were obtained from the Aburaihan Pharmaceutical Company located in Tehran-Iran, which was approved by the quality assurance department of the company. We used ELISA kits (CUSABIO Company, Houston, TX, USA) to analyze the serum levels of NF-kB, follicle-stimulating hormone (FSH), luteinizing hormone $(\mathrm{LH})$, and testosterone in our laboratory (Bahar Toxicology Laboratory, Tehran, Iran).

\section{Experimental model}

The practical model of the experiment is shown in Fig. 1. A total of 25 healthy female rats were randomly selected and divided into 5 groups; 4 test groups and the control. All test subjects were administered oral gavages 7 days, in a volume not exceeding $1 \mathrm{~mL} / 100 \mathrm{~g}$ body weight/rat. Animals from the control group received $0.1 \%$ CMC aqueous solution/day by gavage in the same volume. No significant difference was considered regarding the baseline weights of the animals. Animal body weight changes, food and water consumption, and all clinical signs and symptoms were recorded daily [19].

In the control (vehicle only) group, animals received $0.1 \%$ CMC aqueous solution orally once/day from days 1 to 35 . PCOS groups orally received letrozole at a concentration of $1 \mathrm{mg} / \mathrm{kg}$ dissolved in $0.1 \% \mathrm{CMC}$ solution $(2 \mathrm{~mL} / \mathrm{kg}$ ) once for 21 days [20]. In the second phase of our study, the first PCOS groups received the standard treatment $-20 \mathrm{mcg} /$ $\mathrm{kg} \mathrm{E}+200 \mathrm{mcg} / \mathrm{kg} \mathrm{L}$, dissolved in $0.1 \%$ CMC (2 mL/kg) for 14 days [20]. In the test group, all 5 animals received KRGE at a dose of $100 \mathrm{mg} / \mathrm{kg}$ dissolved in distilled water $(2 \mathrm{~mL} / \mathrm{kg}$ ) [21]. In the combination group, animals received the same doses of KRGE and standard treatment together for the same period of time, and the control group received CMC $0.1 \%$ (14 days).

\section{Hormonal and nuclear factor kappa B assay}

In order to determine the serum concentrations of NF-kB, $\mathrm{FSH}, \mathrm{LH}$, and testosterone, blood samples were collected according to the standard protocol, and the serum was separated by cold centrifuge and collected in microtubes. Hormone levels in the treatment groups were compared with those of the control via chemiluminescence immunoassay method using Cobas E411 from the Roche Company, USA with the serial number 15D3-16 [22,23].

\section{Recovery studies}

A commercial rat serum pool (catalog no. M5905; Sigma Chemical Co., St. Louis, MO, USA) was spiked with various hormone concentrations, and percent recovery and parallel-

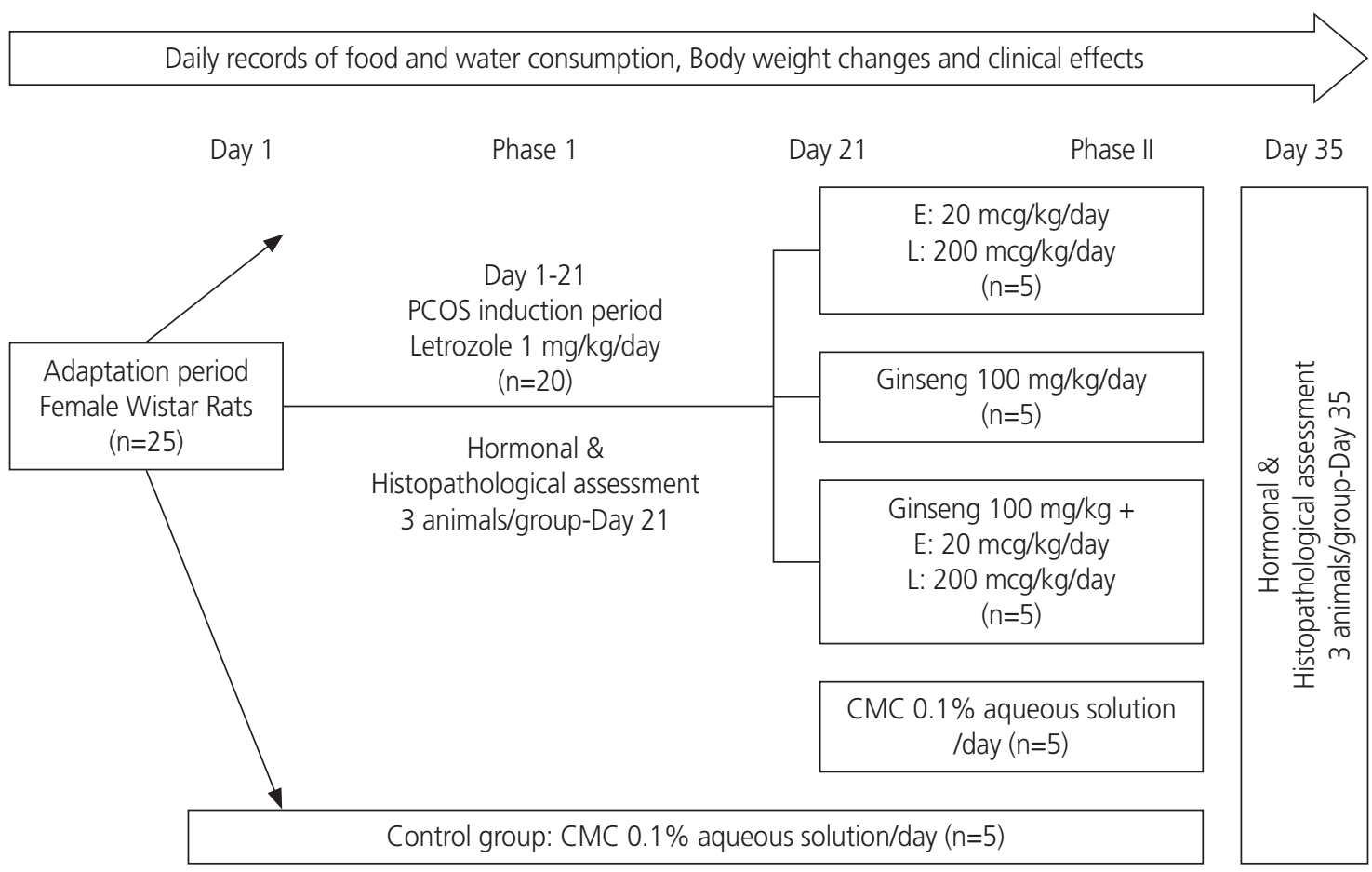

Fig. 1. Experimental model of the study. PCOS, polycystic ovarian syndrome; CMC, carboxymethyl cellulose. 


\section{Obstetrics \& Gynecology Science}

Niknaz Moradi, et al. KRGE in PCOs

ism to the assay standard curve were determined. The serum pool was spiked for each assay, either with hormones across

Table 1. Serum levels of hormones and nuclear factor kappa B (NF-KB) at the end of polycystic ovarian syndrome induction phase (day 21)

\begin{tabular}{lccc}
\hline Variables & CMC $(\mathbf{n}=3)$ & LET $(\mathbf{n = 3})$ & $\boldsymbol{P}$-value \\
\hline LH $(\mathrm{mlU} / \mathrm{mL})$ & $8.446 \pm 0.292$ & $9.576 \pm 0.069$ & $0.020^{\mathrm{a})}$ \\
FSH $(\mathrm{mlU} / \mathrm{mL})$ & $7.606 \pm 0.141$ & $6.833 \pm 0.161$ & $0.024^{\mathrm{a})}$ \\
Testosterone $(\mathrm{ng} / \mathrm{mL})$ & $1.003 \pm 0.067$ & $1.816 \pm 0.073$ & $0.001^{\mathrm{b}}$ \\
NF-kB $(\mathrm{pg} / \mathrm{mL})$ & $12.183 \pm 0.138$ & $16.87 \pm 0.213$ & $0.0001^{\mathrm{c})}$ \\
\hline
\end{tabular}

CMC, carboxymethyl cellulose; LET, letrozole; LH, luteinizing hormone; $\mathrm{FSH}$, follicle-stimulating hormone.

${ }^{\text {a) }} P<0.05$, significant difference; ${ }^{\text {b) }} P<0.01$, moderately significant difference; ${ }^{c} P<0.001$, highly significant difference.

A

$\mathrm{LH}$

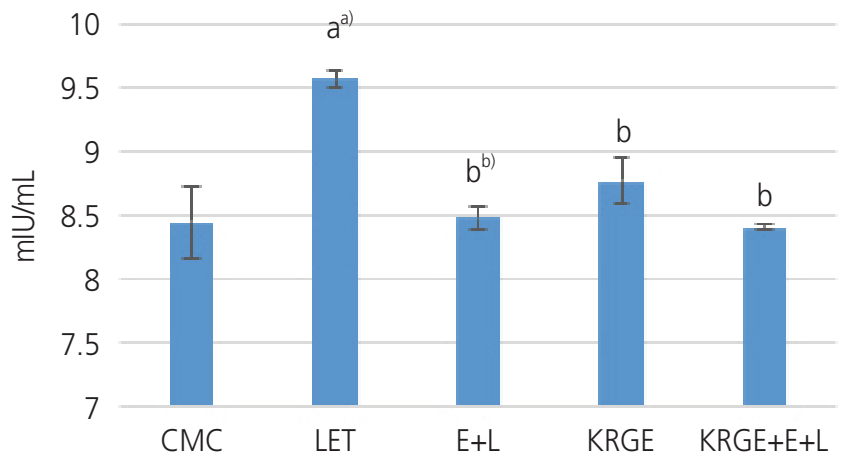

C

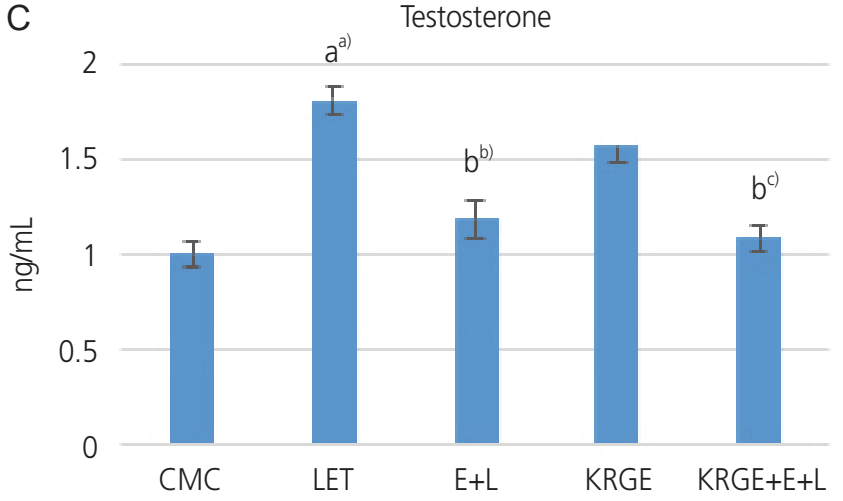

the assay range or vehicle, to determine endogenous hormone levels in the pool. Samples were run in duplicate, and each assay was repeated to confirm results. Hormonal recovery from each spiked sample was determined by subtracting hormone values in vehicle-spiked controls from those in spiked hormone samples.

\section{Histopathological studies}

At the end of the experiments, all rats were anesthetized with ketamine and xylazine, and the dissected ovaries and uteri were placed in a $10 \%$ formalin solution. Samples were sectioned at a thickness of $4 \mu \mathrm{m}$ and stained with hematoxylin and eosin. Follicles containing an oocyte with a nucleus were counted and analyzed by an experienced animal pathologist. In the ovaries, the corpora lutea and follicles at

B

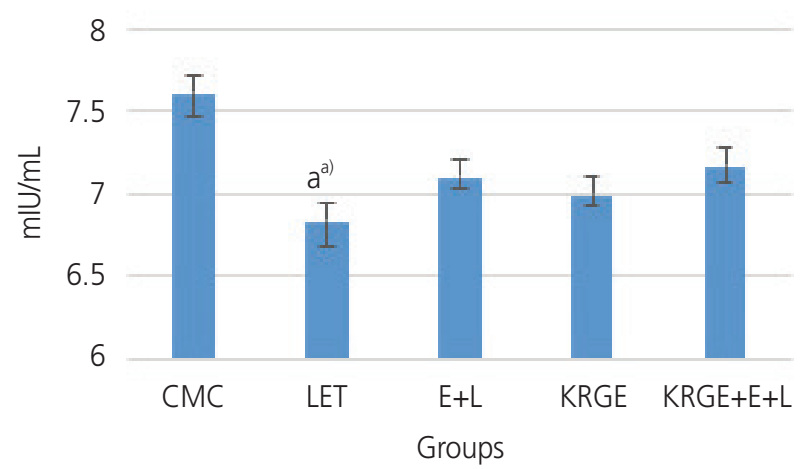

D

$\mathrm{NF}-\mathrm{KB}$

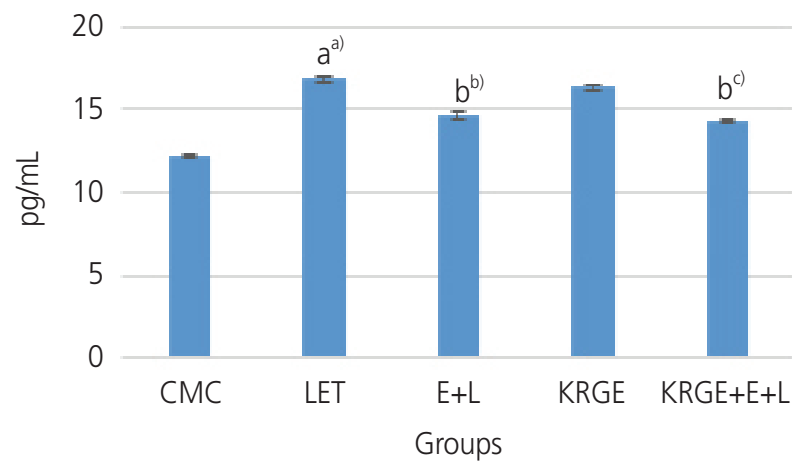

Fig. 2. Serum levels of luteinizing hormone (LH) (A), follicle-stimulating hormone (FSH) (B), testosterone (C) and nuclear factor kappa $B$ (NF-KB), (D) before and after 14 days of administration of standard regimen (ethinyl estradiol $[E]+$ levonorgestrol $[\mathrm{L}]$ ), Korean red ginseng extract (KRGE) monotherapy, and their combinational therapy. (A) shows significant difference in LH levels in treatment groups compared to the levels in control group (carboxymethyl cellulose [CMC]), (B) shows significant difference in FSH levels in other groups compared to letrozole group (letrozole [LET]), (C) shows significant difference in serum testosterone levels in $\mathrm{E}+\mathrm{L}$ and $\mathrm{KRGE}+\mathrm{E}+\mathrm{L}$ groups compared to LET group. (D) shows significant difference in serum NF-KB levels in $E+L$ and $K R G E+E+L$ groups compared to $L E T$ group. Different lowercase letters indicate statistically significant differences between the different groups. ${ }^{a)} P<0.05$, significant difference; ${ }^{b} P<0.01$, moderately significant difference; ${ }^{\mathrm{c}} P<0.001$, highly significant difference. 


\section{Obstetrics \& Gynecology Science}

Vol. 64, No. 3, 2021

different developmental stages were examined using a light microscope (Olympus BX-51; Olympus, Tokyo, Japan) and scored.

\section{Statistical analysis}

In this analysis, treatment and control groups were compared. Values were expressed as means \pm standard deviation (SD). When variances were not significantly different, data were analyzed by the Student's t-test. We used a post hoc test whenever a significant difference between three or more sample means was revealed by an analysis of variance. The level of significance was set at $P<0.05$. All statistical methods were performed by SPSS version 21 (IBM Corp., Armonk, NY, USA).

\section{Results}

\section{Serum analysis}

1) Polycystic ovarian syndrome induction period (day 21)

As demonstrated in Table 1, after the induction of PCOS in this model on day 21, LH levels increased $(P=0.02)$, FSH levels decreased $(P=0.024)$, and testosterone levels increased
$(P=0.001)$ significantly in the letrozole (PCOS) group. Highly significant elevation of NF-KB in the letrozole group also showed the contribution of this inflammatory factor to other hormonal dysregulations in our PCOS model.

\section{2) Treatment period (day 35)}

\section{Luteinizing hormone levels}

As demonstrated in Fig. 2A, all three categories of interventions were able to reduce the serum LH levels to normal levels on day 35 when compared to the levels on day 21 , and all treatments normalized the levels similarly.

\section{Follicle-stimulating hormone levels}

As shown in Fig. 2B, these 3 categories of interventions did not significantly change the FSH levels compared to letrozole. However, FSH changes have no therapeutic value in PCOS.

\section{Testosterone levels}

As shown in Fig. 2C, KRGE monotherapy did not reduce the serum testosterone levels significantly $(1.816 \pm 0.073$ vs. $1.573 \pm 0.148, P=0.099$ ), but its combination with standard treatment could potentiate the antiandrogenic effects of standard treatment.

Table 2. Total body and ovarian weight changes during polycystic ovarian syndrome induction phase (days 1-21)

\begin{tabular}{|c|c|c|c|c|c|}
\hline \multirow{2}{*}{ Groups } & \multicolumn{5}{|c|}{ Total body weight (g) } \\
\hline & Day 1 & Day 21 & $P$-value ${ }^{\text {a) }}$ & $P$-value ${ }^{\text {b) }}$ & $P$-value ${ }^{c}$ \\
\hline Total body weight (g) & & & 1.00 & 0.009 & $<0.001^{d)}$ \\
\hline Control (CMC) & $168.3(5.805)$ & $201.8(11.7)$ & & & \\
\hline Letrozole & $168.8(5.26)$ & $224.2(8.67)$ & & & \\
\hline \multicolumn{6}{|l|}{ Ovarian weight (g) } \\
\hline Control (CMC) & ND & $0.746(0.148)$ & ND & 0.518 & ND \\
\hline Letrozole & & $0.792(0.031)$ & & & \\
\hline \multicolumn{6}{|l|}{ Uterine weight (g) } \\
\hline Control (CMC) & ND & $0.587(0.21)$ & ND & 0.237 & ND \\
\hline Letrozole & & $0.710(0.03)$ & & & \\
\hline \multicolumn{6}{|c|}{ Ovaries weight/whole bodyweight } \\
\hline Control (CMC) & ND & $0.0039 \pm 0.0004$ & ND & 0.845 & ND \\
\hline Letrozole & ND & $0.0034 \pm 0.0000$ & ND & & ND \\
\hline
\end{tabular}

CMC, carboxymethyl cellulose; ND, not determined.

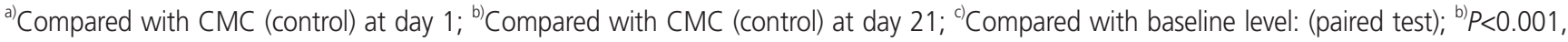
highly significant difference. 


\section{Obstetrics \& Gynecology Science}

Niknaz Moradi, et al. KRGE in PCOs

\section{Nuclear factor kappa B levels}

As described previously, the serum levels of NF-KB were significantly increased in the LET group, compared with the CMC group $(P<0.001)$. KRGE monotherapy did not affect the $\mathrm{NF}-\mathrm{kB}$ serum levels significantly, but its combination therapy $(\mathrm{KRGE}+\mathrm{E}+\mathrm{L})$ caused an marked reduction in serum NF-KB levels.

\section{Animal weights}

1) Total body, ovaries, and uterine weight changes at the induction period (days 1-21)

Table 2 shows the body and organ weights of animals during the induction period. In the beginning, the mean weight of the control group was equal to that of the letrozole group $(168.3 \pm 5.805 \mathrm{~g}$ vs. $168.8 \pm 5.26 \mathrm{~g}, P=1.000)$, but on day 21 , the total body weight of the letrozole group was significantly higher than that of the control group $(201.8 \pm 11.7 \mathrm{~g}$
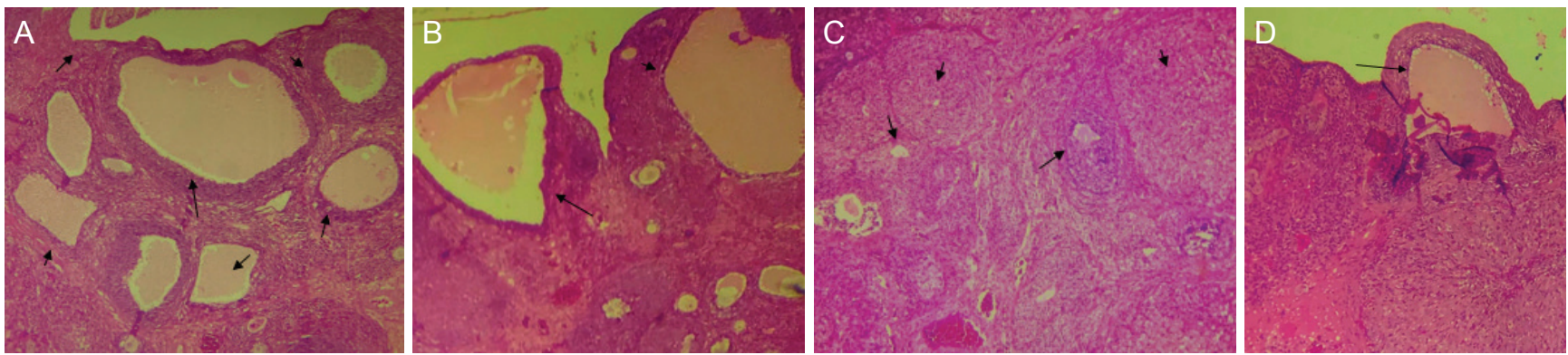

Fig. 3. Histopathological effects of different interventions on ovarian follicular cyst $(\times 100)$. (A) Ovarian tissue of letrozole (LET) group with multiple cystic follicles ( $\times 100)$; (B) Ovarian tissue with prominent follicular cyst $(\times 100)$; (C) Mild ovarian atrophy in Korean red ginseng extract (KRGE) exposed tissues with few follicles (large arrow) and numerous corpus luteum (small arrow). (D) Ovarian tissue of KRGE+ethinyl estradiol (E)+levonorgestrol (L) combination with single follicular cyst (cystic corpora lutea, arrow) $(\times 100)$.

Table 3. Total body and ovarian weight changes during polycystic ovarian syndrome treatment phase (days 21-35)

\begin{tabular}{|c|c|c|c|c|}
\hline Groups $(n=5)$ & Day 21 & Day 35 & $P$-value ${ }^{\text {a) }}$ & $P$-value ${ }^{\text {b) }}$ \\
\hline \multicolumn{5}{|l|}{ Total body weight } \\
\hline Negative control (CMC) & $172.8(8.07)$ & $217(18.01)$ & $0.025^{c)}$ & 0.959 \\
\hline Positive control standard treatment $(\mathrm{E}+\mathrm{L})$ & $202.4(19.7)$ & $217.6(17.9)$ & & \\
\hline KRGE (100 mg/kg) & $198.8(9.65)$ & $228.8(17.56)$ & 0.325 & 0.348 \\
\hline KRGE $(100 \mathrm{mg} / \mathrm{kg})(+\mathrm{E}+\mathrm{L})$ & $190.0(22.7)$ & $208(0.25)$ & 0.535 & 0.508 \\
\hline \multicolumn{5}{|l|}{ Ovaries weight } \\
\hline Negative control (CMC) & $0.587(0.21)$ & $0.322(0.057)$ & 0.518 & $<0.001^{c)}$ \\
\hline Positive control standard treatment $(\mathrm{E}+\mathrm{L})$ & $0.710(0.03)$ & $0.658(0.08)$ & & \\
\hline KRGE (100 mg/kg) & $0.792(0.31)$ & $0.3834(0.41)$ & 0.910 & $<0.001^{c)}$ \\
\hline $\operatorname{KRGE}(100 \mathrm{mg} / \mathrm{kg})(+\mathrm{E}+\mathrm{L})$ & & $0.292(0.113)$ & 0.253 & $<0.001^{c)}$ \\
\hline \multicolumn{5}{|l|}{ Uterine weight } \\
\hline Negative control (CMC) & $0.587(0.21)$ & $0.674(0.140)$ & 0.237 & 0.315 \\
\hline Positive control standard treatment $(\mathrm{E}+\mathrm{L})$ & $0.710(0.03)$ & $0.597(0.077)$ & & \\
\hline KRGE (100 mg/kg) & $237.3 \pm 9.667$ & $0.570(0.16)$ & 0.315 & 0.751 \\
\hline $\operatorname{KRGE}(100 \mathrm{mg} / \mathrm{kg})(+\mathrm{E}+\mathrm{L})$ & $220.3 \pm 12.018$ & $0.564(0.14)$ & 0.609 & 0.661 \\
\hline
\end{tabular}

CMC, carboxymethyl cellulose; E, ethinyl estradiol; L, levonorgestrol; KRGE, Korean red ginseng extract.

${ }^{a}$ Compared with negative control $(\mathrm{CMC})$ at day $35 ;{ }^{\text {b) }}$ Compared with standard treatment $(\mathrm{E}+\mathrm{L})$ at day $35 ;{ }^{\mathrm{c})} \mathrm{P}<0.001$, highly significant difference. 


\section{Obstetrics \& Gynecology Science}

Vol. 64, No. 3, 2021

vs. $224.2 \pm 8.67 \mathrm{~g}, P<0.001)$. The paired student $t$-test also showed highly significant weight gain in the letrozole group $(168.8 \pm 5.26$ vs. $224.2 \pm 8.67, P<0.001)$. Although the mean ovarian and uterine weights and the ratio of ovarian weight to total weight in the letrozole group indicated some inclinations toward change, the differences were not statistically significant. Histopathological analysis showed multiple cysts in the ovaries of the letrozole group (Fig. 3A).

2) Total body weights, ovaries, and uterine weight changes after treatment periods (days 21-35)

\section{Total body weight}

The mean weights of the positive and negative control were relatively similar on day 1 , but the mean body weight of the positive control group was higher than that of the negative control on day $21(P=0.025)$. Notably, the mean body weight of the negative control was in a similar range as that of the $K R G$ and $K R G+E+L$ groups on day 21. After different interventions with standard treatment, the mean ovarian weights in KRGE and $K R G+L+E$ groups were not equal but did not show any statistical significance when compared with the negative control group ( $P>0.05$, Table 3 ). The mean body weights of the standard (OCP) treatment group at day $35(217.6 \pm 19.7 \mathrm{~g})$ were also lower than those of the KRGE group $(237.3 \pm 9.667 \mathrm{~g})$, but the difference was not statistically significant $(P>0.05)$. The same pattern was seen in the KRGE+ standard treatment group (208 [0.25], P>0.05).

\section{Ovaries}

At day 35, the mean ovarian weights of both KRGE-treated groups ( $K R G E$ and $K R G E+E+L$ ) returned to their normal state when compared with those of the negative control group ( $P>0.05)$, whereas those of the standard treatment regimen group was not effective in normalizing the mean ovarian weights at day 35 compared to those of the KRGE-treated groups $(P<0.001)$ (Table 3$)$.

\section{Uteri}

Uterine weight changes showed similar patterns in all experimental groups without any significant difference ( $P>0.05)$.

\section{Histopathological studies of ovaries}

Fig. 3 shows the existence of cystic follicles in the ovaries of female Wistar rats that were exposed to letrozole in a 21 - day oral regimen (Fig. 3A), which reveals the accuracy of this PCOS model. Fig. 3B shows the partial efficacy of the standard regimen $(E+L)$ on reducing the number and size of cysts in treated animals despite abnormal weights of ovaries compared to other groups. As shown in Fig. 3C, mild ovarian atrophy was detected in ovarian tissues of KRGE administered rats with few follicles and numerous corpus luteum ( $\times 100)$. All abnormal cysts disappeared after 14 days of monotherapy with initial positive necropsy results. Ovarian features of $K R G E+E+L$ combination therapy were the same as KRGE monotherapy because the tissue returned to a normal state, one follicular cyst remained, and multiple cysts disappeared.

\section{Discussion}

OCPs are the first-line medications for long-term treatment of reproductive dysfunctions and hyperandrogenism in PCOS patients, but they may cause cardiovascular disorders, insulin resistance, and other adverse health effects, especially in susceptible women $[2,4]$. Based on this concept and previous clinical experiences on the safe uses of KRGE as a successful alternative medicine in pre- and post-menopausal women for improving their sexual life $[24,25]$, we decided to compare the effects of KRGE monotherapy and its combination with the first-line treatment in a letrozole-induced PCOS model and compare the clinical, hormonal, inflammatory and histopathological evidence with standard (first line) treatment $(E+L)$. Notably, our necropsy analysis found the efficacy of KRGE in controlling all histological signs of PCOS by normalizing the excessive ovarian weights, reducing LH levels in serum, and removing the ovarian cysts in all treated animals of the letrozole-induced rat model, which mimics the main histological and biochemical features of PCOS in women [20]. As shown in Table 2 and Fig. 2A, letrozole increased the total body weight and ovarian weight without any change in uterine weights. However, 14 days of KRGE oral regimen and its combination with standard treatment normalized the letrozole-induced pathological changes. This finding supports the hypothesis about the successful therapeutic roles of KRGE treatment in PCOS, especially in combination with a standard regimen, based on the additional antiandrogenic and antiinflammatory effects, which were reported for the first time and is the most important achievement of our present study.

Letrozole is an aromatase inhibitor that increases the tes- 


\section{Obstetrics \& Gynecology Science}

Niknaz Moradi, et al. KRGE in PCOs

tosterone levels and induces hyperandrogenism in animal and human exposures $[17,20]$. The serum testosterone level is one of the main diagnostic and prognostic biomarkers in PCOS [1]. The present model showed the accuracy, sensitivity, and specificity of this biomarker, which was significantly increased during the PCOS induction period $(P<0.01$; Fig. 2C) and was reduced to a normal state by the standard treatment $(P<0.01)$. Unfortunately, KRGE monotherapy did not show any significant effect on serum testosterone levels. Therefore, the antiandrogenic effects of KRGE were not significant enough to be detected: however, in combination with a standard regimen, antiandrogenic effects were significant $(P<0.01)$. Notably, the average serum levels of testosterone was $1.086 \pm 0.06 \mathrm{ng} / \mathrm{mL}$ in the combination group and $1.186 \pm 0.09 \mathrm{ng} / \mathrm{mL}$ in the standard treatment group; hence, these data suggest the potential role of KRGE in helping the standard regimen control serum testosterone levels. We suggest further assessment of the antiandrogenic potencies of this combinational regimen in PCOS patients who develop hyperandrogenism at adjusted doses over long-term periods.

Many recent studies have shown the inflammatory mechanisms in the pathogenesis of PCOS $[25,26]$ and the key role of NF-KB in the disease's progression $[13,27]$. Recent studies show the anti-inflammatory properties of ginsenosides as the main constituents of ginseng by suppressing the production of pro-inflammatory cytokines and regulating the activities of the inflammatory signaling pathway of NF-KB [28]. The present study did not confirm the effects of KRGE monotherapy on the serum levels of NF-kB, but KRGE intensified the antiinflammatory effects of the standard regimen with the KRGE+OCP-combination model $(P<0.01)$. We observed the potential role of KRGE in enhancing the anti-inflammatory effects of the standard treatment because the serum levels of NF-KB decreased more with the combination model $(14.25 \pm 0.06 \mathrm{pg} / \mathrm{mL}$ compared to $14.63 \pm 0.2 \mathrm{pg} / \mathrm{mL}$, Fig. 2D). Further clinical and immunohistochemical studies are mandated to confirm this valuable preclinical achievement.

The other aspect of this study was the proposed effect of KRGE on LH serum levels; as shown in Fig. $2 A$ and $B, 14$ days of orally administering KRGE reduced $L H$ serum levels $(P<0.05)$ significantly without any practical effect on serum FSH levels. $\mathrm{Rg} 1$ is one of the main and specific ginsenosides of KRGE, which mediates some of the steroidal effects of KRGE by binding to the estrogen receptor and sharing many protective actions of estrogen in various physiologi- cal systems [20]. Ginseng in ovariectomized mice caused a significant reduction in LH serum levels, which clearly confirms these estrogenic effects [29], and this result also suggests the differential effect of KRGE on LH levels in PCOS. In addition, the combination model of KRGE and standard treatment showed a better synergistic effect on normalizing the $\mathrm{LH}$ levels because the average serum level of $\mathrm{LH}$ in the combination group was $8.41 \pm 0.03 \mathrm{mlU} / \mathrm{mL}$, whereas the average serum level of $\mathrm{LH}$ in the standard treatment group was $8.48 \pm 0.09 \mathrm{mlU} / \mathrm{mL}$. Further clinical studies are warranted to confirm these preliminary findings in our animal setting.

This model did not cause any endometrial hyperplasia; however, any change in uterine weights and the histopathology of the uterus, significant ovarian weight gain, ovarian hyperplasia, and the formation of numerous cysts were the main symptoms of PCOS in this study, which was in accordance to previous animal evidence $[1,14]$ as shown in Fig. 2A. Although the standard regimen reduced the number of cysts in the treated animals and caused partial removal of hyperplasia, ovarian weights remained practically unchanged after 14 days. However, KRGE administration alone caused a significant decrease in the ovarian weights after 14 days by inducing ovarian atrophy and removing all ovarian cysts (Fig. 2C). Histopathological findings also confirmed the efficacy of KRGE in normalizing the ovarian weights and removing the ovarian cysts with sufficient hormonal effects. The same pattern was observed in the KRGE+OCPs group with additional anti-inflammatory and antiandrogenic effects.

The present study assumed that KRGE has beneficial effects on the clinical, hormonal, and histopathological PCOS symptoms, especially when it is co-administered with a standard OCP regimen over 14 days. The 14-day continuous monotherapy using a $100 \mathrm{mg} / \mathrm{kg} /$ day regimen reduced the LH serum levels and significantly atrophied rat ovaries and removed the cysts from the ovaries of treated rats. KRGE alone was, however, unable to control the testosterone and NF$\mathrm{KB}$ levels; consequently, we could suggest it as an excellent alternative medicine in PCOS patients. Further studies are recommended in preclinical and clinical models to confirm the promising effects of KRGE in controlling the symptoms of PCOS. 


\section{Obstetrics \& Gynecology Science}

Vol. 64, No. 3, 2021

\section{Acknowledgments}

We are thankful to the Islamic Azad University, Tehran Medical Sciences University (IAUTMU), for the financial and technical support of this study. We also acknowledge Dr. Hasti Azar Abad as the veterinary pathologist of the present study for her excellent support.

\section{Conflict of interest}

No potential conflict of interest relevant to this article was reported.

\section{Ethical approval}

The Ethics Committee of Islamic Azad University, Tehran Medical Sciences University (IAUTMU), approved the protocols of this study under the number IR.IAU.TMU. REC.1397.313, and the animals were cared for in accordance with the Guidelines for Animal Experiments of the Ministry of Health and Medical Education of Iran for the Care and Use of Laboratory Animals and the CCAC Guidelines for Care and Use of Experimental Animals.

\section{Patient consent}

None.

\section{Funding information}

None.

\section{References}

1. Walters KA, Gilchrist RB, Ledger WL, Teede HJ, Handelsman DJ, Campbell RE. New perspectives on the pathogenesis of PCOS: neuroendocrine origins. Trends Endocrinol Metab 2018;29:841-52.

2. Yildiz BO. Oral contraceptives in polycystic ovary syndrome: risk-benefit assessment. Semin Reprod Med
2008;2:111-20.

3. Victor VM, Rocha M, Bañuls $C$, Sanchez-Serrano $M$, Sola E, Gomez M, et al. Mitochondrial complex I impairment in leukocytes from polycystic ovary syndrome patients with insulin resistance. J Clin Endocrinol Metab 2009;94:3505-12.

4. Teede HJ, Misso ML, Deeks AA, Moran LJ, Stuckey BG, Wong $\mathrm{J}$, et al. Assessment and management of polycystic ovary syndrome: summary of an evidence-based guideline. Med J Aust 2011;195:S65-112.

5. Damone AL, Joham AE, Loxton D, Earnest A, Teede HJ, Moran LJ. Depression, anxiety and perceived stress in women with and without PCOS: a community-based study. Psychol Med 2019;49:1510-20.

6. Behboodi Moghadam Z, Fereidooni B, Saffari M, Montazeri $A$. Measures of health-related quality of life in PCOS women: a systematic review. Int J Womens Health 2018; 10:397-408.

7. Palioura E, Diamanti-Kandarakis E. Polycystic ovary syndrome (PCOS) and endocrine disrupting chemicals (EDCs). Rev Endocr Metab Disord 2015;16:365-71.

8. Zuo T, Zhu M, Xu W, Wang Z, Song H. Iridoids with genipin stem nucleus inhibit lipopolysaccharide-induced inflammation and oxidative stress by blocking the NF-kB pathway in polycystic ovary syndrome. Cell Physiol Biochem 2017;43:1855-65.

9. Escobar-Morreale HF, Luque-Ramírez M, González F. Circulating inflammatory markers in polycystic ovary syndrome: a systematic review and metaanalysis. Fertil Steril 2011;95:1048-58.e1.

10. Liu M, Gao J, Zhang Y, Li P, Wang H, Ren X, et al. Serum levels of TSP-1, NF-KB and TGF- $\beta 1$ in polycystic ovarian syndrome (PCOS) patients in northern China suggest PCOS is associated with chronic inflammation. Clin Endocrinol (Oxf) 2015;83:913-22.

11. Nehir Aytan A, Bastu E, Demiral I, Bulut H, Dogan M, Buyru F. Relationship between hyperandrogenism, obesity, inflammation and polycystic ovary syndrome. Gynecol Endocrinol 2016;32:709-13.

12. Sirmans SM, Weidman-Evans E, Everton V, Thompson D. Polycystic ovary syndrome and chronic inflammation: pharmacotherapeutic implications. Ann Pharmacother 2012;46:403-18.

13. Zaafan MA, Abdelhamid AM, Ibrahim SM. The protective effect of Korean red ginseng against rotenone- 


\section{Obstetrics \& Gynecology Science}

Niknaz Moradi, et al. KRGE in PCOs

induced Parkinson's disease in rat model: modulation of nuclear factor- $\mathrm{k} \beta$ and caspase-3. Curr Pharm Biotechnol 2019;20:588-94.

14. Kim SJ, Kwak HJ, Kim DS, Choi HM, Sim JE, Kim SH, et al. Protective mechanism of Korean red ginseng in cisplatin-induced ototoxicity through attenuation of nuclear factor-kB and caspase-1 activation. Mol Med Rep 2015;12:315-22.

15. Choi JH, Jang M, Nah SY, Oh S, Cho IH. Multitarget effects of Korean Red Ginseng in animal model of Parkinson's disease: antiapoptosis, antioxidant, antiinflammation, and maintenance of blood-brain barrier integrity. J Ginseng Res 2018;42:379-88.

16. Shim MK, Lee YJ. Estrogen receptor is activated by Korean red ginseng in vitro but not in vivo. J Ginseng Res 2012;36:169-75.

17. Najaf Najafi M, Ghazanfarpour M. Effect of phytoestrogens on sexual function in menopausal women: a systematic review and meta-analysis. Climacteric 2018;21:437-45.

18. Korea Ginseng Corp. Korean red ginseng extract, ginseng root, tea, capsule [Internet]. Seou (KR): Ginseng Corp.; c2020 [cited 2019 Oct 31]. Available from: https://www.kgcus.com/.

19. Jung JH, Park HT, Kim T, Jeong MJ, Lim SC, Nah SY, et al. Therapeutic effect of Korean red ginseng extract on infertility caused by polycystic ovaries. J Ginseng Res 2011;35:250-5.

20. Kafali H, Iriadam M, Ozardali I, Demir N. Letrozoleinduced polycystic ovaries in the rat: a new model for cystic ovarian disease. Arch Med Res 2004;35:103-8.

21. Fernández-Martínez E, Pérez-Soto E, González-Hernández C, Ortiz Ml, Pérez-Álvarez V, Muriel P, et al. Immunomodulatory effects by oral contraceptives in normal and cholestatic female rats: role of cytokines. Int Immunopharmacol 2014;21:10-9.

22. Mahsa S, Naeimeh D, Hamed DP, Hossein K, Nazanin SJ. Comparison of ginseng extract and metformin on improvement of polycystic ovary syndrome (POS). J Entomol Zool Stud 2015;3:501-5.

23. Baravalle C, Salvetti NR, Mira GA, Pezzone N, Ortega $\mathrm{HH}$. Microscopic characterization of follicular structures in letrozole-induced polycystic ovarian syndrome in the rat. Arch Med Res 2006;37:830-9.

24. Chung HS, Hwang I, Oh KJ, Lee MN, Park K. The effect of Korean red ginseng on sexual function in premenopausal women: placebo-controlled, double-blind, crossover clinical trial. Evid Based Complement Alternat Med 2015;2015:913158.

25. Oh KJ, Chae MJ, Lee HS, Hong HD, Park K. Effects of Korean red ginseng on sexual arousal in menopausal women: placebo-controlled, double-blind crossover clinical study. J Sex Med 2010;7:1469-77.

26. Sun $Y F$, Zhang $X$, Wang $X Y$, Jia W. Effect of long-term intake of ginseng extracts on gut microbiota in rats. Zhongguo Zhongyao Zazhi 2018.43:3927-32.

27. González F, Rote NS, Minium J, Kirwan JP. Increased activation of nuclear factor kappaB triggers inflammation and insulin resistance in polycystic ovary syndrome. J Clin Endocrinol Metab 2006;91:1508-12.

28. Koc O, Ozdemirici S, Acet M, Soyturk U, Aydin S. Nuclear factor-kB expression in the endometrium of normal and overweight women with polycystic ovary syndrome. J Obstet Gynaecol 2017;37:924-30.

29. Xu Y, Ding J, Ma XP, Ma YH, Liu ZQ, Lin N. Treatment with Panax ginseng antagonizes the estrogen decline in ovariectomized mice. Int J Mol Sci 2014;15:7827-40. 